Journal of Nonlinear Mathematical Physics

\title{
On the Geodesic Flow on the Group of Diffeomorphisms of the Circle with a Fractional Sobolev Right-Invariant Metric
}

Marcus Wunsch

To cite this article: Marcus Wunsch (2010) On the Geodesic Flow on the Group of Diffeomorphisms of the Circle with a Fractional Sobolev Right-Invariant Metric, Journal of Nonlinear Mathematical Physics 17:1, 7-11, DOI:

https://doi.org/10.1142/S1402925110000544

To link to this article: https://doi.org/10.1142/S1402925110000544

Published online: 04 January 2021 


\title{
ON THE GEODESIC FLOW ON THE GROUP OF DIFFEOMORPHISMS OF THE CIRCLE WITH A FRACTIONAL SOBOLEV RIGHT-INVARIANT METRIC
}

\author{
MARCUS WUNSCH \\ Research Institute for Mathematical Sciences \\ Kyoto University \\ Kyoto 606-8502, Japan \\ mwunsch@kurims.kyoto-u.ac.jp
}

Received 29 June 2009

Accepted 9 September 2009

\begin{abstract}
We show that the geodesic flow on the infinite-dimensional group of diffeomorphisms of the circle, endowed with a fractional Sobolev metric at the identity, is described by the generalized ConstantinLax-Majda equation with parameter $a=-\frac{1}{2}$.
\end{abstract}

Keywords: Geodesic flow; fractional Sobolev metric; generalized CLM equation.

Mathematics Subject Classification: 53D25, 35Q35

\section{Introduction: The Dual Generalized Constantin-Lax-Majda Equation}

The generalized Constantin-Lax-Majda equation with parameter $a \in \mathbb{R} \cup\{+\infty\}$

$$
\left\{\begin{array}{l}
\omega_{t}+a v \omega_{x}=\omega v_{x}, \quad x \in \mathbb{S}:=\mathbb{R} / 2 \pi \mathbb{Z}, t>0 \\
v_{x}(t, x)=\mathcal{H} \omega(t, x) \\
\omega(0, x)=\omega^{0}(x)
\end{array}\right.
$$

(henceforth referred to as the $g C L M$ equation) was first derived in [17]. Here, the (spatial) Hilbert transform of a function $f$, denoted above by $\mathcal{H} f$, is defined via the Fourier transform $\mathcal{F}$ by $\mathcal{F}(\mathcal{H} f)(\xi)=-\sqrt{-1} \operatorname{sign}(\xi) \mathcal{F}(f)(\xi), \xi \in \mathbb{S}$. Thus, $\mathcal{H}$ gives rise to an $L^{2}(\mathbb{S})$-isometry, and $\mathcal{H}^{2} f=-f$.

For different values of $a \in \mathbb{R}$, the $g C L M$ equation interpolates between several onedimensional model equations arising in fluid dynamics. For example, if $a=0$, the $g C L M$ equation reduces to the classical Constantin-Lax-Majda equation [10] mimicking the 3D vorticity equation. A model for the quasi-geostrophic equations [11] is obtained by setting $a=-1$, and if $a=1$, we obtain the De Gregorio equation [13,14]. For the first two cases, [10] and [11] proved that many smooth initial data give rise to solutions which blow up in finite time, while [17] provided strong numerical evidence for global existence of 
solutions for the case $a=1$. Moreover, if $a=\infty$ (the case of which bears close resemblance to the $2 \mathrm{D}$ vorticity equation) it was shown analytically in [17] that solutions exist for all times.

Let us define the dual generalized Constantin-Lax-Majda equation ( $g C L M^{*}$ equation for short) with parameter $a^{*} \in \mathbb{R} \cup\{-\infty\}$ via

$$
\left\{\begin{array}{l}
\omega_{t}-v \omega_{x}=a^{*} \omega v_{x}, \quad x \in \mathbb{S}, t>0 \\
v_{x}(t, x)=\mathcal{H} \omega(t, x) \\
\omega(0, x)=\omega^{0}(x)
\end{array}\right.
$$

It can obviously be recovered from the $g C L M$ equation with parameter $a \in \mathbb{R}$ by $\omega(t, x) \mapsto$ $\omega\left(a^{*} t, x\right)$, where $a^{*}=-\frac{1}{a}$ (if $a \rightarrow 0$, so that $a^{*} \rightarrow-\infty$, we arrive at the CLM equation in the limit).

\section{The Geodesic Flow on $\mathcal{D}(\mathbb{S})$ Endowed with the Right-invariant $H^{\frac{1}{2}}(\mathbb{S})$ Metric}

In this paper, we observe that the $g C L M^{*}$ equation with parameter $a^{*}=2$ describes the geodesic flow on the infinite-dimensional Lie group of orientation-preserving diffeomorphisms of the circle $\mathbb{S}, \mathcal{D}(\mathbb{S})$, endowed with the $H^{\frac{1}{2}}(\mathbb{S})$ fractional Sobolev right-invariant metric given at the identity by

$$
\langle u, v\rangle_{i d}=\int \Lambda u v d x, \quad u, v \in T_{i d} \mathcal{D}(\mathbb{S}) \equiv \mathcal{C}^{\infty}(\mathbb{S})
$$

where $\Lambda f$ is defined via the Fourier transform $\mathcal{F}$ :

$$
\mathcal{F}(\Lambda f)(\xi)=\xi \operatorname{sign}(\xi) \mathcal{F}(f)(\xi), \quad \xi \in \mathbb{S}
$$

Consequently, we have the calculation rules $\mathcal{H} \Lambda f=\Lambda \mathcal{H} f=-f_{x}$, and $\Lambda f=\mathcal{H} f_{x}$.

To define a smooth right-invariant Riemannian metric on $\mathcal{D}(\mathbb{S})$, we extend the inner product (2.1) to each tangent space $T_{\varphi} \mathcal{D}(\mathbb{S})$ by right translation:

$$
\langle V, W\rangle_{\varphi}=\left\langle V \circ \varphi^{-1}, W \circ \varphi^{-1}\right\rangle_{i d} \quad \text { for } V, W \in T_{\varphi} \mathcal{D}(\mathbb{S})
$$

The existence of a covariant derivative $\nabla$ preserving the inner product (2.2), which is necessary to derive the geodesic equation of the metric, is guaranteed by the following theorem.

Theorem 2.1 [9]. Consider a non-degenerate continuous inner product $\langle.,$.$\rangle on T_{i d} \mathcal{D}(\mathbb{S}) \equiv$ $\mathcal{C}^{\infty}(\mathbb{S})$, and extend it to each tangent space $T_{\varphi} \mathcal{D}(\mathbb{S})$ by right translation. If there exists a bilinear operator $B: \mathcal{C}^{\infty}(\mathbb{S}) \times \mathcal{C}^{\infty}(\mathbb{S}) \rightarrow \mathcal{C}^{\infty}(\mathbb{S})$ such that

$$
\langle B(u, v), w\rangle=\langle u,[v, w]\rangle \quad u, v, w \in \mathcal{C}^{\infty}(\mathbb{S})
$$

where the commutator [., .] is given by $[v, w]=v w_{x}-v_{x} w$, then there is a unique Riemannian connection $\nabla$ on $\mathcal{D}(\mathbb{S})$ associated with the right-invariant metric $\langle.,$.$\rangle .$ 
The geodesic equation (also referred to as the Euler equation) is now given as $[1,2,9]$

$$
u_{t}=B(u, u)
$$

Let us first determine the bilinear form $B(u, v)$ using formula $(2.3)$ :

$$
\begin{aligned}
\langle u,[v, w]\rangle & =\int_{\mathbb{S}} \Lambda u v w_{x} d x-\int_{\mathbb{S}} \Lambda u v_{x} w d x \\
& =\int_{\mathbb{S}} \mathcal{H}\left(\mathcal{H} u_{x} v\right) \mathcal{H} w_{x} d x-\int_{\mathbb{S}} \mathcal{H}\left(\mathcal{H} u_{x} v_{x}\right) \mathcal{H} w d x \\
& =\int_{\mathbb{S}} \mathcal{H}\left(\mathcal{H} u_{x} v\right) \Lambda w d x+\int_{\mathbb{S}} \partial_{x}^{-1} \mathcal{H}\left(\mathcal{H} u_{x} v_{x}\right) \Lambda w d x
\end{aligned}
$$

hence

$$
B(u, v)=\mathcal{H}\left(\mathcal{H} u_{x} v\right)+\partial_{x}^{-1} \mathcal{H}\left(\mathcal{H} u_{x} v_{x}\right),
$$

and so the geodesic equation reads

$$
u_{t}=\mathcal{H}\left(u \mathcal{H} u_{x}\right)+\partial_{x}^{-1} \mathcal{H}\left(u_{x} \mathcal{H} u_{x}\right) .
$$

Differentiation with respect to the space variable $x$ now yields

$$
u_{t x}=\mathcal{H}\left(u \mathcal{H} u_{x x}+u_{x} \mathcal{H} u_{x}\right)+\mathcal{H}\left(u_{x} \mathcal{H} u_{x}\right)=\mathcal{H}\left(u \mathcal{H} u_{x x}\right)+2 \mathcal{H}\left(u_{x} \mathcal{H} u_{x}\right)
$$

Applying the Hilbert transform $\mathcal{H}$ to this equation, we see that

$$
\mathcal{H} u_{t x}=-u \mathcal{H} u_{x x}-2 u_{x} \mathcal{H} u_{x}
$$

If we set $\omega=\mathcal{H} u_{x}$, then we obtain the $g C L M^{*}$ equation with parameter $a^{*}=2$ :

$$
\omega_{t}=2 \omega v_{x}+v \omega_{x}, \quad v_{x}=\mathcal{H} \omega .
$$

Thus we have proven

Theorem 2.2. The generalized Constantin-Lax-Majda equation (1.1) with parameter $a=$ $-\frac{1}{2}$ (or equivalently, the gCLM* equation (1.2) with parameter $a^{*}=2$ ) corresponds to the equation of the geodesic flow on $\mathcal{D}(\mathbb{S})$ with respect to the right-invariant metric (2.1), (2.2).

Remark 2.1. It is important to point out that while we have proven that geodesics must obey the evolution prescribed by the $g C L M^{*}$ equation with parameter $a^{*}=2$, we have not demonstrated the existence of geodesics on the manifold $\mathcal{D}(\mathbb{S})$ endowed with the $H^{\frac{1}{2}}(\mathbb{S})$ right-invariant metric. This will be proven in a more detailed analysis, together with results about related topics on the $g C L M^{*}$ equation and the geometry of $\mathcal{D}(\mathbb{S})$ endowed with the fractional Sobolev metric.

Let us also mention that our considerations are not in the least limited to the periodic case: appropriate conditions at infinity (cf. $[5,6]$ ) ensuring that the diffeomorphisms approach the identity far out should facilitate the study of the case on the line $\mathbb{R}$.

Remark 2.2. Recently, there has been written a host of articles (cf. [7-9, 15, 16] and the references therein) dedicated to the study of differential geometric features of $\mathcal{D}(\mathbb{S})$ endowed 
with several right-invariant metrics, among which the $L^{2}(\mathbb{S}), H^{1}(\mathbb{S})$, and (the homogeneous) $\dot{H}^{1}(\mathbb{S})$ metrics attracted the greatest attention, since in these cases, the geodesic equations are re-expressions of the Burgers [3], Camassa-Holm [4], and Hunter-Saxton equations [12], respectively. Thus it is of interest to notice that also the fractional Sobolev metric can give rise to a physically meaningful equation, lying "between" the Burgers equation and the Hunter-Saxton equation, both of which have solutions which lose their initial regularity in finite time $[9,18]$.

Remark 2.3. In the hierarchy of the generalized Constantin-Lax-Majda equation [17], the equation discussed lies between the 1D model equation for the quasi-geostrophic equation [11] and the original CLM equation [10]. Solutions to both equations are known to become singular in most cases: This, together with the above remark, supports the conjecture that solutions to the $g C L M^{*}$ equation with parameter $a^{*}=2$ blow up in finite time as well. We will address this question in a forthcoming study as well.

\section{Acknowledgments}

This study was inspired by the ideas of [9]. The author would like to thank Professor Adrian Constantin for his encouraging comments and beneficial advice. He is also grateful for constructive suggestions and important references provided by the anonymous referee.

\section{References}

[1] V. Arnold, Sur la géometrie différentielle des groupes de Lie de dimension infinie et ses application à l'hydrodynamique des fluides parfaits, Ann. Inst. Grenoble 16 (1966) 319-361.

[2] V. Arnold and B. Khesin, Topological Methods in Hydrodynamics (Springer, New York, 1998).

[3] J. Burgers, A mathematical model illustrating the theory of turbulence, Adv. Appl. Mech. 1 (1948) 177-199.

[4] R. Camassa and D. Holm, An integrable shallow water equation with peaked solitons, Phys. Rev. Lett. 71 (1993) 1661-1664.

[5] M. Cantor, Perfect fluid flows over $\mathbb{R}^{n}$ with asymptotic conditions, J. Funct. Anal. 18 (1975) $73-84$.

[6] A. Constantin, Existence of permanent and breaking waves for a shallow water equation: a geometric approach, Ann. Inst. Fourier 50 (2000) 321-362.

[7] A. Constantin and B. Kolev, Geodesic flow on the diffeomorphism group of the circle, Comment. Math. Helv. 78 (2003) 787-804.

[8] A. Constantin and B. Kolev, Integrability of invariant metrics on the diffeomorphism group of the circle, J. Nonlinear Sci. 16 (2006) 109-122.

[9] A. Constantin and B. Kolev, On the geometric approach to the motion of inertial mechanical systems, J. Phys. A: Math. Gen. 35 (2002) R51-R79.

[10] P. Constantin, P. Lax and A. J. Majda, A simple one-dimensional model for the threedimensional vorticity equation, Comm. Pure Appl. Math. 38 (1985) 715-724.

[11] A. Córdoba, D. Córdoba and M. A. Fontelos, Integral inequalities for the Hilbert transform applied to a nonlocal transport equation, J. Math. Pure Appl. 86 (2006) 529-540.

[12] J. K. Hunter and R. Saxton, Dynamics of director fields, SIAM J. Appl. Math. 51 (1991) $1498-1521$.

[13] S. De Gregorio, On a one-dimensional model for the three-dimensional vorticity equation, J. Stat. Phys. 59 (1990) 1251-1263.

[14] S. De Gregorio, A partial differential equation arising in a 1D model for the 3D vorticity equation, Math. Methods Appl. Sci. 19 (1996) 1233-1255. 
[15] J. Lenells, The Hunter-Saxton equation: a geometric approach, SIAM J. Math. Anal. 40 (2008) 266-277.

[16] J. Lenells, The Hunter-Saxton equation describes the geodesic flow on a sphere, J. Geom. Phys. 57 (2007) 2049-2064.

[17] H. Okamoto, T. Sakajo and M. Wunsch, On a generalization of the Constantin-Lax-Majda equation, Nonlinearity 21 (2008) 2447-2461.

[18] Zh. Yin, On the structure of solutions to the periodic Hunter-Saxton equation, SIAM J. Math. Anal. 36 (2004) 272-283. 